\title{
Influence of Multiwalled Carbon Nanotubes and Biostimulators on Growth and Content of Bioactive Constituents of Karkade (Hibiscus sabdariffa L.)
}

\author{
Layth Sareea Al-Rekaby \\ Department of Biology, College of Science, University of Al-Qadisiyah, Qadisiyyah, Iraq \\ Correspondence should be addressed to Layth Sareea Al-Rekaby; layth.sareea@qu.edu.iq
}

Received 22 August 2017; Revised 14 December 2017; Accepted 19 December 2017; Published 11 February 2018

Academic Editor: Shamsul Hayat

Copyright (C) 2018 Layth Sareea Al-Rekaby. This is an open access article distributed under the Creative Commons Attribution License, which permits unrestricted use, distribution, and reproduction in any medium, provided the original work is properly cited.

\begin{abstract}
This work was carried out to evaluate the effect of different concentrations of multiwalled carbon nanotubes (MWCNT), 0, 500, and $1000 \mathrm{mg} / \mathrm{L}$, and biostimulators (Delfan plus), 0 and $10 \mathrm{ml} / \mathrm{L}$, and their interactions (sprayed on the plant shoot and soil until the complete wetness) on growth and production of bioactive constituents of karkade (Hibiscus sabdariffa L.) planted in plastic pots. After finishing experiment, growth parameters (length of shoot and root, number of leaves, branches, and fruits, chlorophyll content, and dry weight of calyces yield) were measured and also the active compounds of aqueous extracts calyces were measured by Gas Chromatography-Mass Spectrometry (GC-MS). The obtained results explained that the experimental treatment caused a significant increase in all measured parameters. GC-MS analysis shows quantitative and qualitative alterations of bioactive constituents of water extract from calyxes of karkade, where plant production of 60 active compounds at the combination of $1000 \mathrm{mg} / \mathrm{L}$ of MWCNT and $10 \mathrm{ml} / \mathrm{L}$ of Delfan plus was compared to untreated plants which produced 4 only.
\end{abstract}

\section{Introduction}

Medical plants researches have been expanded apparently during the last decade and international trend towards the use of natural plant remedies increased the need for more information about the properties and uses of medicinal plants. Hibiscus sabdariffa L., commonly known as roselle (English) and karkade (Arabic), belongs to Malvaceae and is an important annual crop grown successfully in subtropical and tropical climates [1]. The calyxes of karkade are rich in bioactive compounds including organic acids, phenolic compounds, vitamin A and vitamin C [2], flavonoid (anthocyanins), gossypetin, quercetin, pectin [3], saponins, glycosides, and alkaloids [4]. Additionally, Nnam and Onyeke [5] mentioned that calyxes contain $6.4 \%$ protein, $79.25 \%$ carbohydrates, $5.13 \%$ fat, $2.7 \%$ crude fiber, and $6.52 \%$ ash. Fresh or dried calyxes of karkade have been used traditionally as herbal drinks (cold and hot) because karkade has different therapeutic properties such as anticancer, antiviral, antifungal, antibacterial, antioxidant, antidiabetic, antihypertensive, antihyperlipidemia (anticholesterol), antiobesity, antispasmodic, and antitumor effects, decreasing the viscosity of the blood and stimulating intestinal peristalsis, and can be used to treat sore throats, coughs, and genital problems and it has also nephro/hepatoprotective and renal/diuretic effect and so forth [6].

In the recent years, there has been an increasing interest in nanoagriculture; the use of nanotechnology in agriculture and field application are highly important and are widely used in agricultural sciences, especially in the fields of biotechnology and tissue culture. The uptake of carbon nanomaterials by plants has shown a very recent field of nanoagriculture. Nanotubes are capable of interacting with biomolecules and creating functional nanosystems for transportation of other materials within cells, which leads to interaction of nanotubes and other compounds at morphological, cellular, and even molecular levels [7]. The application of biostimulators for different plants has positive effect on plant development due to containing high levels of free amino acids, total nitrogen, organic matter, and organic carbon [8]. 
TABLE 1: Characteristics of multiwalled carbon nanotubes (MWCNT).

\begin{tabular}{lc}
\hline Aspect & Powder \\
Colour & Black \\
Length & $10-50 \mu \mathrm{m}$ \\
Outer diameter & $8-15 \mathrm{~nm}$ \\
Inside diameter & $3-5 \mathrm{~nm}$ \\
Specific surface area & $233 \mathrm{~m}^{2} / \mathrm{g}$ \\
Purity & $>95 \mathrm{wt} \%$ \\
True density & $\sim 2.1 \mathrm{~g} / \mathrm{cm}^{3}$ \\
Bulk density & $0.15 \mathrm{~g} / \mathrm{cm}^{3}$ \\
Ash & $<1.5 \mathrm{wt} \%$ \\
Electrical conductivity & $>100 \mathrm{~s} / \mathrm{cm}$ \\
\hline
\end{tabular}

TABLE 2: Characteristics of biostimulators (Delfan plus).

\begin{tabular}{lccc}
\hline Physical-chemical characteristics & \multicolumn{2}{c}{ Chemical analysis } \\
\hline Aspect & Liquid & Free amino acids & $24.00 \%$ \\
Colour & Brown & Total nitrogen $(\mathrm{N})$ & $9.00 \%$ \\
Density & $1.2 \mathrm{~g} / \mathrm{cc}$ & Organic matter & $37.00 \%$ \\
$\mathrm{pH}$ & 7.2 & Organic carbon & $23.00 \%$ \\
\hline
\end{tabular}

Therefore, in the view of the medicinal importance of karkade calyxes, this work aimed to evaluate the effect of different concentrations of multiwalled carbon nanotubes and biostimulators (Delfan plus) on growth and quantitative and qualitative alterations of bioactive constituents measured by GC-MS.

\section{Materials and Methods}

A pot experiment was conducted in Biology Department, College of Science, University of Al-Qadisiyah, which included planting seeds of karkade plant in 18 plastic pots (5 seeds per pot) on 15/02/2016, and then the developing plants were reduced to one plant per pot. The experimental treatment was sprayed on the plant shoot and soil until the complete wetness; multiwalled carbon nanotubes (MWCNT) (Table 1) were added at three concentrations, 0 , 500 , and $1000 \mathrm{mg} / \mathrm{L}$, on 10/05/2016. Biostimulators (Delfan plus) (Table 2) were added at two concentrations of 0 and $10 \mathrm{ml} / \mathrm{L}$ on $15 / 5 / 2016$. The addition of two factors was repeated after one month, respectively.

After finishing the experiment (01/11/2016), growth parameters were measured, including length of shoot and root, number of leaves, branches, and fruits, dry weight of calyxes yield, chlorophyll content (measured by chlorophyll meter 502 SPAD), and the content of bioactive constituents per plant after preparation of aqueous extract of the calyxes of karkade harvested during periods of experience and then those bioactive constituents were measured by GC-MS technique (manufacturer: Shimadzu, GCMS-QP2010 Ultra system comprising a AOC-20i, Japan), according to the method of Srinivasan et al. [9].
2.1. Statistical Analysis. Data analysis was carried out using Randomized Complete Blocks Design (RCBD) in a factorial arrangement $(3 \times 2)$ with three replications per each treatment. Revised Least Significant Difference (RLSD) was used to compare treatment means at probability level of 0.05 when treatment effect was significant [10].

\section{Results}

The statistical analysis of Table 3 elucidates that increasing concentrations of both multiwalled carbon nanotubes (MWCNT) and biostimulators (Delfan plus) added to the plants alone or with each other lead to a significant increase in all studied parameters of karkade (length of shoot and root, number of leaves, branches, and fruits, chlorophyll content, dry weight of calyxes yield, and number of bioactive constituents) in comparison with those obtained from untreated plants; using the combination of $1000 \mathrm{mg} / \mathrm{L}$ MWCNT and $10 \mathrm{ml} / \mathrm{L}$ Delfan plus caused the highest increase which reached $30.47 \%$ length of shoot, $45.73 \%$ length of root length, $64.89 \%$ number of leaves, $62.08 \%$ number of branches, $60.98 \%$ number of fruits, chlorophyll content of $13.77 \%$, and $48.30 \%$ dry weight of calyxes yield compared with untreated plants.

The GC-MS analysis of water extract from calyxes of karkade showed that untreated plants contain 4 bioactive constituents: 3,7,11,15-tetramethyl-2-hexadecen-1-ol; 1,1-bicyclohexyl, 2-(2-methylpropyl)-trans; 3-buten-2-one,4(2-hydroxy-2,6,6-trimethylcyclohexyl); and 1,2-benzenedicarboxylic acid, diisooctyl ester (Table 4). 3-Buten-2-one, 4-(2-hydroxy-2,6,6-trimethylcyclohexyl) was identified as a major chemical constituent and gave the highest peak and relative area depending on the GC-MS chromatogram that showed 4 peaks (Figure 1).

Karkade treated without adding MWCNT and $10 \mathrm{ml} / \mathrm{L}$ Delfan plus produced 13 bioactive constituents (Table 5). Acetic acid was identified as a major chemical constituent depending on the GC-MS chromatogram that showed 13 peaks, while thujone gave the highest peak (Figure 2).

Result in Table 6 explained that karkade treated with $500 \mathrm{mg} / \mathrm{L}$ MWCNT and without adding Delfan plus produced 12 bioactive constituents. Depending on the GC-MS chromatogram in Figure 3, 1,2-benzenedicarboxylic acid, mono (2-ethylhexyl) ester gave the highest relative area and peak compared to other compounds.

The treatment of karkade with a combination of $500 \mathrm{mg} / \mathrm{L}$ MWCNT and $10 \mathrm{ml} / \mathrm{L}$ Delfan plus showed 47 bioactive constituents (Table 7). Menthol, 1-(butyn-3-one-1-yl)-(1R,2S,5R) showed the highest relative area and peak compared to other compounds depending on the GC-MS chromatogram (Figure 4).

Karkade treated with $1000 \mathrm{mg} / \mathrm{L}$ MWCNT and without adding Delfan plus produced 41 bioactive constituents (Table 8). 1,2-Benzenedicarboxylic acid, diisooctyl ester gave the highest relative area and peak compared to other compounds depending on the GC-MS chromatogram (Figure 5). Finally, karkade treated with a combination of $100 \mathrm{mg} / \mathrm{L}$ MWCNT and $10 \mathrm{ml} / \mathrm{L}$ Delfan plus showed 60 bioactive constituents (Table 9). Depending on the GC-MS 
TABLE 3: Significant effect of multiwalled carbon nanotubes and biostimulators (Delfan plus) in the studied characteristics of Hibiscus sabdariffa L.

\begin{tabular}{|c|c|c|c|c|c|c|c|c|c|}
\hline \multirow{2}{*}{$\begin{array}{l}\text { Multiwalled carbon } \\
\text { nanotubes } \\
\text { concentration }(\mathrm{mg} / \mathrm{L})\end{array}$} & \multirow{2}{*}{$\begin{array}{l}\text { Biostimulators } \\
\text { (Delfan plus) } \\
\text { concentration } \\
(\mathrm{ml} / \mathrm{L})\end{array}$} & \multicolumn{2}{|c|}{ Length of $(\mathrm{cm})$} & \multicolumn{3}{|c|}{ Number of (per plant) } & \multirow{2}{*}{$\begin{array}{l}\text { Chlorophyll } \\
\text { content } \\
\text { (SPAD) }\end{array}$} & \multirow{2}{*}{$\begin{array}{c}\text { DW of calyx } \\
\text { yield } \\
\text { (gm/fruit) }\end{array}$} & \multirow{2}{*}{$\begin{array}{l}\text { Number of } \\
\text { bioactive } \\
\text { constituents } \\
\text { (per plant) }\end{array}$} \\
\hline & & Shoot & Root & Leaves & Branches & Fruits & & & \\
\hline \multirow{2}{*}{0} & 0 & 51.14 & 18.13 & 15.63 & 6.62 & 36.22 & 51.55 & 24.52 & 4 \\
\hline & 10 & 57.44 & 22.43 & 23.33 & 11.42 & 44.13 & 53.42 & 29.72 & 13 \\
\hline \multirow{2}{*}{500} & 0 & 52.13 & 23.54 & 25.31 & 8.32 & 57.81 & 54.82 & 31.61 & 12 \\
\hline & 10 & 64.81 & 26.22 & 33.14 & 14.52 & 69.11 & 56.19 & 36.62 & 47 \\
\hline \multirow{2}{*}{1000} & 0 & 59.73 & 28.73 & 29.46 & 10.74 & 77.54 & 57.22 & 40.41 & 41 \\
\hline & 10 & 73.55 & 33.41 & 44.52 & 17.46 & 92.83 & 59.78 & 47.43 & 60 \\
\hline \multicolumn{2}{|c|}{ RLSD at 0.05} & 9.55 & 2.47 & 8.69 & 4.63 & 10.61 & 2.33 & 5.51 & 7.97 \\
\hline
\end{tabular}

TABLE 4: Bioactive constituents of water extract of calyxes of karkade (Hibiscus sabdariffa L.) from combination treatment without multiwalled carbon nanotubes and biostimulators (Delfan plus).

\begin{tabular}{lcccccc}
\hline & & & \multicolumn{3}{c}{ Peak report TIC } \\
Peak & R. time & Area & Area\% & Height & Height $\%$ & Name \\
\hline 1 & 12.62 & 93268 & 10.28 & 19921 & 24.88 & 3,7,11,15-Tetramethyl-2-hexadecen-1-ol \\
2 & 13.56 & 70006 & 7.72 & 12624 & 15.77 & 1,1' -Bicyclohexyl, 2-(2-methylpropyl)-, trans- \\
3 & 18.57 & 689870 & 76.07 & 35869 & 44.80 & 3-Buten-2-one, 4-(2-hydroxy-2,6,6-trimethylcyclohexyl)- \\
4 & 20.42 & 53697 & 5.92 & 11659 & 14.56 & 1,2-Benzenedicarboxylic acid, diisooctyl ester \\
& & 906841 & 100.00 & 80073 & 100.00 & \\
\hline
\end{tabular}

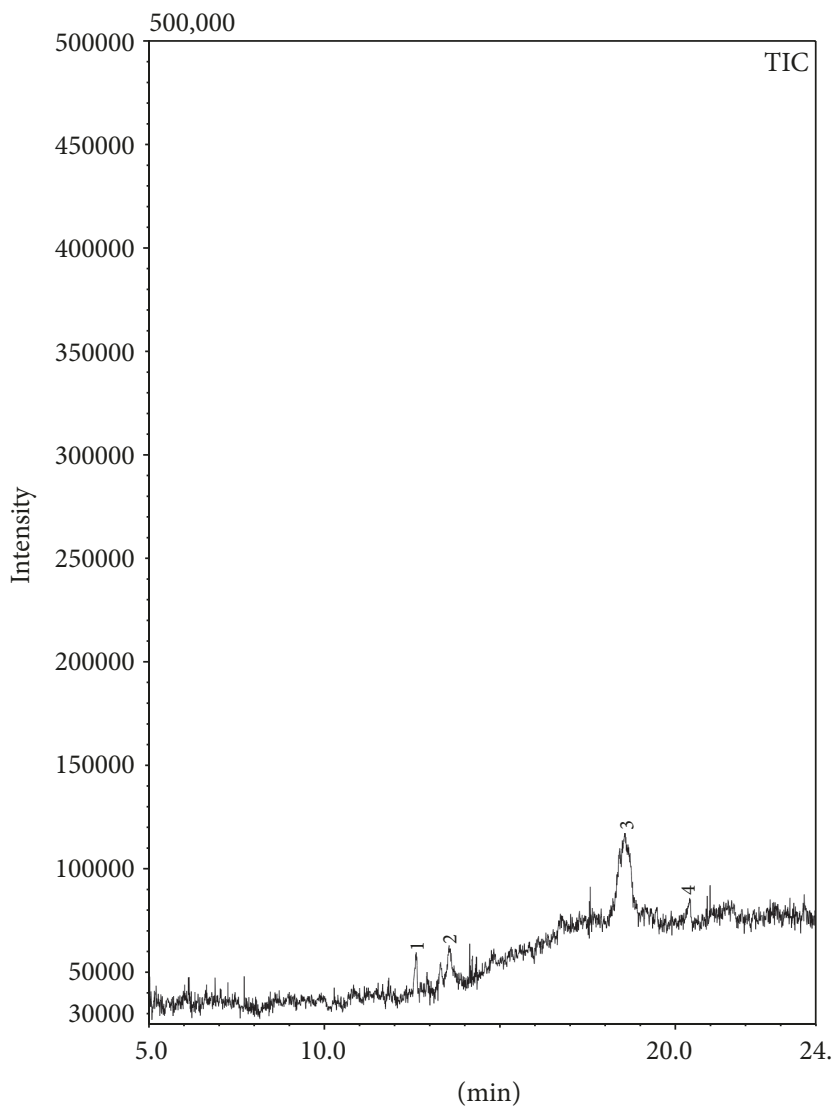

FIGURE 1: GC-MS chromatogram of water extract of calyxes of karkade (Hibiscus sabdariffa L.) from combination treatment without multiwalled carbon nanotubes and biostimulators (Delfan plus).

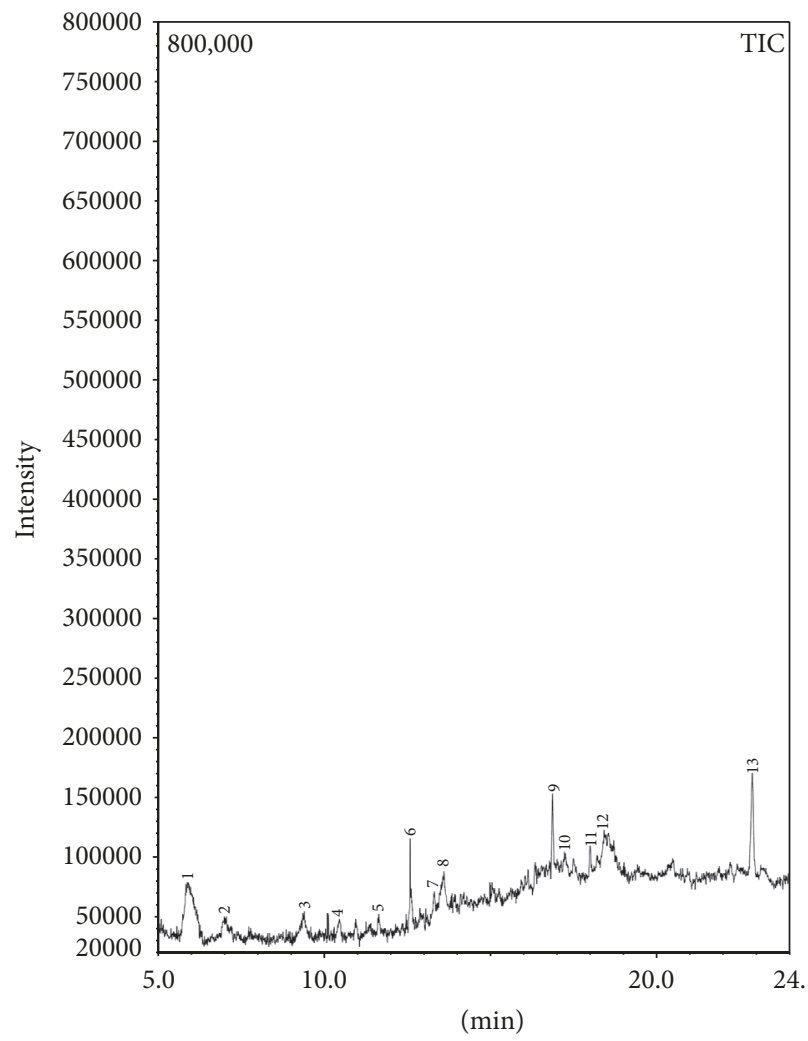

FIGURE 2: GC-MS chromatogram of water extract of calyxes of karkade (Hibiscus sabdariffa L.) from combination treatment without multiwalled carbon nanotubes and $10 \mathrm{ml} / \mathrm{L}$ biostimulators (Delfan plus). 
TABLE 5: Bioactive constituents of water extract of calyxes of karkade (Hibiscus sabdariffa L.) from combination treatment without multiwalled carbon nanotubes and $10 \mathrm{ml} / \mathrm{L}$ biostimulators (Delfan plus).

\begin{tabular}{lccccccc}
\hline & & & & & & Peak report TIC \\
Peak & R. time & Area & Area\% & Height & Height\% & Name \\
\hline 1 & 5.87 & 924451 & 28.07 & 44787 & 9.86 & Acetic acid \\
2 & 6.98 & 234129 & 7.11 & 18401 & 4.05 & Camphor \\
3 & 9.39 & 264901 & 8.04 & 23849 & 5.25 & Bicyclo[2.2.1]heptane, 2-methoxy-1,7,7-trimethyl- \\
4 & 10.45 & 66366 & 2.01 & 13395 & 2.95 & 2-Butenoic acid, 2-methyl-, (Z)- \\
5 & 11.63 & 58361 & 1.77 & 16685 & 3.67 & 1-Hexadecanol \\
6 & 12.58 & 237929 & 7.22 & 78034 & 17.18 & 3,7,11,15-Tetramethyl-2-hexadecen-1-ol \\
7 & 13.31 & 45794 & 1.39 & 14885 & 3.28 & 1,3-Cyclopentadiene, 5,5-dimethyl-2-ethyl- \\
8 & 13.60 & 200938 & 6.10 & 28416 & 6.26 & Diazoacetic acid, 2-isopropyl-5-methylcyclohexyl ester \\
9 & 16.87 & 187103 & 5.68 & 65937 & 14.52 & 3,6-Octadien-1-ol, 3,7-dimethyl-, (Z)- \\
10 & 17.22 & 96494 & 2.93 & 17373 & 3.82 & 3-Oxabicyclo[4.1.0]heptan-2-one, 4,4,7,7-tetramethyl- \\
11 & 18.00 & 72451 & 2.20 & 23296 & 5.13 & 12-Oxabicyclo[9.1.0]dodeca-3,7-diene, 1,5,5,8-tetramethyl-, [1R-(1R $\left.{ }^{*}, 3 \mathrm{E}, 7 \mathrm{E}, 11 \mathrm{R}^{*}\right)$ ]- \\
12 & 18.42 & 487748 & 14.81 & 27530 & 6.06 & 3-Buten-2-one, 4-(2-hydroxy-2,6,6-trimethylcyclohexyl)- \\
13 & 22.88 & 416944 & 12.66 & 81663 & 17.98 & Thujone \\
\hline
\end{tabular}

TABLE 6: Bioactive constituents of water extract of calyxes of karkade (Hibiscus sabdariffa L.) from combination treatment of 500 mg/L multiwalled carbon nanotubes and without biostimulators (Delfan plus).

\begin{tabular}{|c|c|c|c|c|c|c|}
\hline \multicolumn{7}{|c|}{ Peak report TIC } \\
\hline Peak & R. time & Area & Area\% & Height & Height $\%$ & Name \\
\hline 1 & 5.10 & 786921 & 11.31 & 30384 & 4.41 & 2-Pentanone, 4-hydroxy-4-methyl- \\
\hline 2 & 14.29 & 1861443 & 26.75 & 84961 & 12.34 & 2(3H)-Benzofuranone, hexahydro-3a,7a-dimethyl-, cis- \\
\hline 3 & 15.90 & 210700 & 3.03 & 35279 & 5.12 & Tritetracontane \\
\hline 4 & 16.94 & 349173 & 5.02 & 37447 & 5.44 & Docosyl pentafluoropropionate \\
\hline 5 & 17.63 & 11414 & 0.16 & 4964 & 0.72 & Tetratriacontane \\
\hline 6 & 17.73 & 48075 & 0.69 & 15669 & 2.28 & Tetratetracontane \\
\hline 7 & 17.81 & 41981 & 0.60 & 15121 & 2.20 & Tritetracontane \\
\hline 8 & 19.17 & 820233 & 11.79 & 115136 & 16.72 & Tetratriacontane \\
\hline 9 & 20.48 & 2197954 & 31.59 & 278588 & 40.46 & 1,2-Benzenedicarboxylic acid, mono(2-ethylhexyl) ester \\
\hline 10 & 21.56 & 141100 & 2.03 & 16363 & 2.38 & Eicosane, 2-cyclohexyl- \\
\hline 11 & 22.29 & 127819 & 1.84 & 23936 & 3.48 & 1-Heneicosanol \\
\hline \multirow[t]{2}{*}{12} & 23.36 & 360897 & 5.19 & 30782 & 4.47 & cis-13,16-Docasadienoic acid \\
\hline & & 6957710 & 100.00 & 688630 & 100.00 & \\
\hline
\end{tabular}

chromatogram in Figure 6, Naphthalenol,decahydro-1,4a dimethyl-7-(1-methylidene)-,[1R(1.alpha.,4a.beta.,8a.alpha.)] showed the highest relative area and peak compared to other compounds.

\section{Discussions}

On the basis of previous results, the reasons for significant increasing growth indicators by adding factor are due to the fact that multiwalled carbon nanotubes (MWCNT) have sizes ranging from 8 to $15 \mathrm{~nm}$ (Table 1) and a large amount of surface area in relation to mass and thus have a high ability to penetrate the walls of plant cells and act as a smart transport system in the plant as well as multiple walls increasing the surface area of the mass; these results are consistent with Canas et al. [11] on the carrot, cabbage, lettuce, cucumber, and onion, Khodakovskaya et al. [12] on the tomato, and Heydari [13] on the anthurium. 
TABLE 7: Bioactive constituents of water extract of calyxes of karkade (Hibiscus sabdariffa L.) from combination treatment of 500 mg/L multiwalled carbon nanotubes and $10 \mathrm{ml} / \mathrm{L}$ biostimulators (Delfan plus).

\begin{tabular}{|c|c|c|c|c|c|c|}
\hline & & & & & & Peak report TIC \\
\hline Peak & R. time & Area & Area\% & Height & Height $\%$ & Name \\
\hline 1 & 8.82 & 141226 & 0.94 & 14453 & 0.41 & Borneol \\
\hline 2 & 9.46 & 162535 & 1.09 & 20610 & 0.58 & $\begin{array}{l}\text { 1H-Benzocycloheptene, } 2,4 \mathrm{a}, 5,6,7,8,9,9 \mathrm{a} \text {-octahydro-3,5,5-trimethyl-9-methylene-, } \\
\text { (4aS-cis)- }\end{array}$ \\
\hline 3 & 9.93 & 248143 & 1.66 & 19212 & 0.54 & 2-Butenoic acid, 2-methyl-, (E)- \\
\hline 4 & 10.48 & 481302 & 3.22 & 80512 & 2.28 & Dodecan-1-yl acetate \\
\hline 5 & 11.60 & 129429 & 0.87 & 20711 & 0.59 & Cyclopropanecarboxylic acid, 1-hydroxy-, (2,6-di-t-butyl-4-methylphenyl) ester \\
\hline 6 & 12.35 & 108383 & 0.72 & 28348 & 0.80 & 3,7,11,15-Tetramethyl-2-hexadecen-1-ol \\
\hline 7 & 12.64 & 269674 & 1.80 & 79065 & 2.24 & 3,7,11,15-Tetramethyl-2-hexadecen-1-ol \\
\hline 8 & 13.00 & 244025 & 1.63 & 86620 & 2.45 & 3,7,11,15-Tetramethyl-2-hexadecen-1-ol \\
\hline 9 & 13.33 & 293411 & 1.96 & 63445 & 1.80 & $(-)-$ Spathulenol \\
\hline 10 & 13.57 & 194491 & 1.30 & 30385 & 0.86 & 2-Pentadecanone, 6,10,14-trimethyl- \\
\hline 11 & 13.83 & 67118 & 0.45 & 19221 & 0.54 & 2-Naphthalenemethanol, 1,2,3,4,4a,5,6,7-octahydro- $\alpha, \alpha, 4 \mathrm{a}, 8$-tetramethyl-, (2R-cis)- \\
\hline 12 & 14.66 & 45902 & 0.31 & 17959 & 0.51 & Veridiflorol \\
\hline 13 & 14.76 & 93078 & 0.62 & 34590 & 0.98 & $\begin{array}{c}\text { 2-Naphthalenemethanol, 2,3,4,4a,5,6,7,8-octahydro- } \alpha, \alpha, 4 \mathrm{a}, 8 \text {-tetramethyl-, } \\
\text { [2R-(2.alpha.,4a.be)] }\end{array}$ \\
\hline 14 & 14.83 & 175845 & 1.18 & 81537 & 2.31 & Hexadecanoic acid, ethyl ester \\
\hline 15 & 15.06 & 275082 & 1.84 & 28540 & 0.81 & Hexadecanoic acid, ethyl ester \\
\hline 16 & 15.22 & 411108 & 2.75 & 109720 & 3.10 & 1-Hexadecen-3-ol, 3,5,11,15-tetramethyl- \\
\hline 17 & 15.68 & 400775 & 2.68 & 89433 & 2.53 & Tricyclo[5.2.2.0(1,6)] undecan-3-ol, 2-methylene-6,8,8-trimethyl- \\
\hline 18 & 15.83 & 83819 & 0.56 & 31391 & 0.89 & Limonen-6-ol, pivalate \\
\hline 19 & 16.04 & 141274 & 0.94 & 67137 & 1.90 & 2(4H)-Benzofuranone, 5,6,7,7a-tetrahydro-4,4,7a-trimethyl- \\
\hline 20 & 16.09 & 351866 & 2.35 & 191370 & 5.42 & Longipinocarveol, trans- \\
\hline 21 & 16.36 & 116915 & 0.78 & 48041 & 1.36 & Longipinocarveol, trans- \\
\hline 22 & 16.44 & 53494 & 0.36 & 20051 & 0.57 & 10-Methyltricyclo[4.3.1.1(2,5)]undecan-10-ol \\
\hline 23 & 16.78 & 159689 & 1.07 & 61966 & 1.75 & Succinic acid, ethyl non-5-yn-3-yl ester \\
\hline 24 & 16.88 & 78982 & 0.53 & 33741 & 0.95 & Bicyclo[2.2.1]heptan-2-ol, 4-chloro-1,7,7-trimethyl-, exo- \\
\hline 25 & 16.96 & 111111 & 0.74 & 40230 & 1.14 & Dodecanoic acid \\
\hline 26 & 17.12 & 209656 & 1.40 & 76583 & 2.17 & 9,12-Octadecadienoic acid, ethyl ester \\
\hline 27 & 17.30 & 58891 & 0.39 & 24040 & 0.68 & 9,12,15-Octadecatrienoic acid, (Z,Z,Z)- \\
\hline 28 & 17.58 & 67358 & 0.45 & 18877 & 0.53 & Phytol \\
\hline 29 & 17.78 & 63878 & 0.43 & 21067 & 0.60 & 1,2-Benzenedicarboxylic acid, diisooctyl ester \\
\hline 30 & 18.00 & 309133 & 2.07 & 120882 & 3.42 & 4-(2,2-Dimethyl-6-methylenecyclohexyl)butanal \\
\hline 31 & 18.58 & 48232 & 0.32 & 21779 & 0.62 & Hexadecanoic acid, 2-methylpropyl ester \\
\hline 32 & 18.66 & 149865 & 1.00 & 64467 & 1.82 & Z,Z-8,10-Hexadecadien-1-ol \\
\hline 33 & 18.94 & 181596 & 1.21 & 51056 & 1.44 & n-Hexadecanoic acid \\
\hline 34 & 19.37 & 244278 & 1.63 & 81298 & 2.30 & Heptanoic acid \\
\hline 35 & 19.49 & 1286488 & 8.60 & 423334 & 11.98 & Citronellyl tiglate \\
\hline 36 & 19.65 & 186885 & 1.25 & 53306 & 1.51 & Bicyclo[2.2.1]heptan-2-ol, 2,3,3-trimethyl- \\
\hline 37 & 19.82 & 54252 & 0.36 & 18932 & 0.54 & 4-Methyldocosane \\
\hline 38 & 20.13 & 68098 & 0.46 & 24263 & 0.69 & 1-Eicosene \\
\hline 39 & 20.44 & 971615 & 6.50 & 98698 & 2.79 & 1-Cyclohexanone, 2-methyl-2-(3-methyl-2-oxobutyl) \\
\hline 40 & 20.62 & 122710 & 0.82 & 32654 & 0.92 & 3-Buten-2-ol, 4-(2,6,6-trimethyl-2-cyclohexen-1-yl)- \\
\hline 41 & 20.82 & 177718 & 1.19 & 43055 & 1.22 & (1S-(1Alpha,2alpha,4beta))-1-isopropenyl-4-methyl-1,2-cyclohexanediol \\
\hline 42 & 20.96 & 885998 & 5.92 & 264130 & 7.47 & $2 \mathrm{H}$-Indeno[1,2-b]furan-2-one, 3,3a,4,5,6,7,8,8b-octahydro-8,8-dimethyl \\
\hline 43 & 21.54 & 753783 & 5.04 & 95420 & 2.70 & 1-(3-Methoxymethyl-2,5,6-trimethylphenyl)ethanone \\
\hline
\end{tabular}


TABLE 7: Continued.

\begin{tabular}{ccccccc}
\hline & & & & & \multicolumn{2}{c}{ Peak report TIC } \\
\hline 44 & 22.20 & 370247 & 2.48 & 48444 & 1.37 & Name \\
45 & 22.88 & 304991 & 2.04 & 43927 & 1.24 & Fumaric acid, ethyl 2-methylallyl ester \\
46 & 23.13 & 252921 & 1.69 & 27372 & 0.77 & 7-Hexadecenal, (Z) \\
47 & 23.72 & 3349386 & 22.39 & 561901 & 15.90 & Cyclopropanemethanol,. alpha.,2-dimethyl-2-(4-methyl-3-pentenyl)-, \\
& & & & & [1.alpha.(R ${ }^{*}$ ),2.alpha.]- \\
& & & & &
\end{tabular}

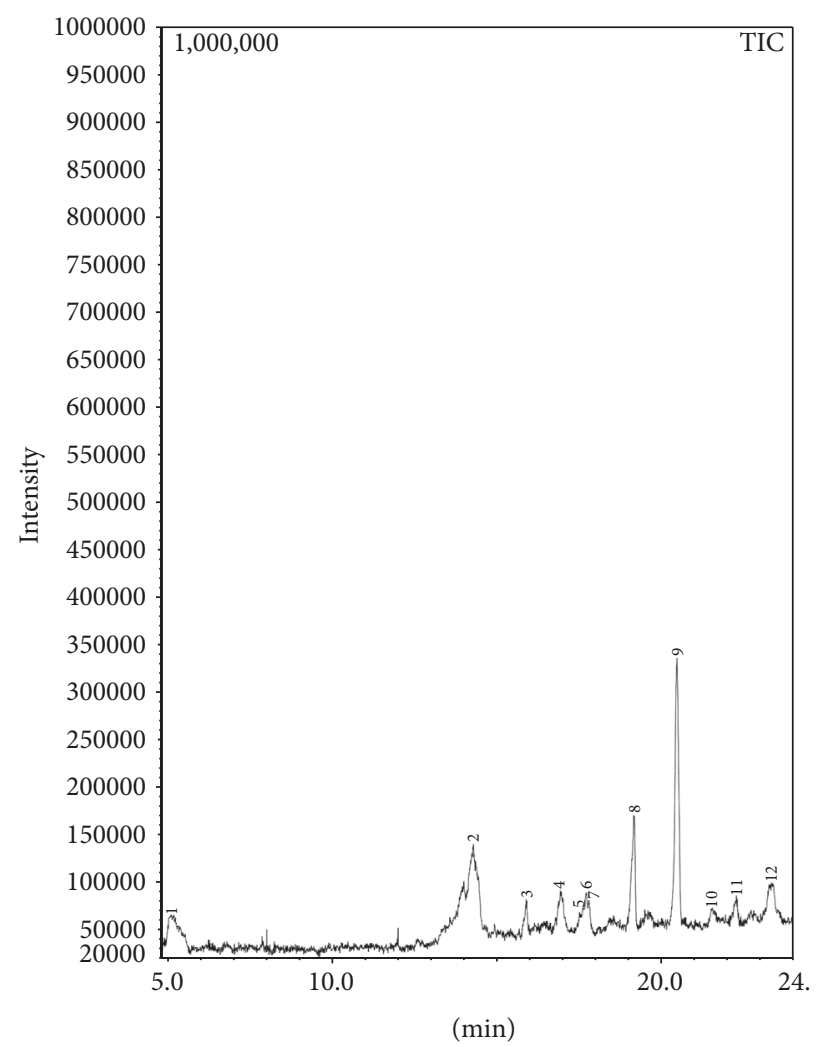

FIGURE 3: GC-MS chromatogram of water extract of calyxes of karkade (Hibiscus sabdariffa L.) from combination treatment of 500 mg/L multiwalled carbon nanotubes and without biostimulators (Delfan plus).

Extensive research has shown that the penetration of MWCNT into the plant cells can bring about changes in metabolic activity, leading to an increase in biomass. The tubular nanoparticle is easy to transport inside the plant. MWCNT increased water uptake through changes in the lipid formation, rigidity, and permeability of cell membrane and also enhanced net assimilation of $\mathrm{CO}_{2}$. MWCNT might act as regulators for growth or could regulate the marker genes to enhance cell culture growth by increasing cell wall formation, cell divisions, and water transport [14, 15]. MWCNT can penetrate cell wall of leaves and induce growth of plants and development (enhancement of shoot length and root length). They also could organize nutrients absorption in the plant such as increased nitrogen, potassium, calcium, and phosphorus while decreasing sodium percent and as a result increasing total chlorophyll [16].
Additionally, MWCNT can be introduced in the plant cell by their attachment to carrier proteins, through aquaporins, using ionic channels, by endocytosis, and through their merging with organic compounds. In the particular case of MWCNT, their entrance is associated mainly with the creation of new pores in the cells [17]. The characteristics are unique of MWCNT (nanoparticle size, ability to penetrate cells, high biochemical reactivity, and rapid distribution inside plant system) and make them an attractive tool for crop management techniques. In this respect, specific types of nanoparticles in low doses have not been found to be harmful to plants but instead are capable of activating specific physiological processes, promoting photosynthesis and nitrogen metabolism, and therefore improving the growth of the plants [18]. They also play a key role in cell wall reinforcement during plant development, cell elongation, 
TABLE 8: Bioactive constituents of water extract of calyxes of karkade (Hibiscus sabdariffa L.) from combination treatment of $1000 \mathrm{mg} / \mathrm{L}$ multiwalled carbon nanotubes and without biostimulators (Delfan plus).

\begin{tabular}{|c|c|c|c|c|c|c|}
\hline & & & & & & Peak report TIC \\
\hline Peak & R. time & Area & Area\% & Height & Height\% & Name \\
\hline 1 & 7.02 & 765601 & 2.17 & 56572 & 0.86 & Bicyclo[2.2.1]heptan-2-one, 1,7,7-trimethyl-, (1S)- \\
\hline 2 & 8.74 & 82130 & 0.23 & 15029 & 0.23 & Pentanoic acid, 4-methyl- \\
\hline 3 & 8.87 & 81976 & 0.23 & 10541 & 0.16 & 1,6,10-Dodecatriene, 7,11-dimethyl-3-methylene- \\
\hline 4 & 9.38 & 520061 & 1.47 & 49940 & 0.76 & Isoborneol \\
\hline 5 & 9.87 & 32654 & 0.09 & 10191 & 0.16 & Caryophyllene \\
\hline 6 & 9.91 & 9809 & 0.03 & 5883 & 0.09 & Geranyl ethyl ether 2 \\
\hline 7 & 10.44 & 1145521 & 3.24 & 132334 & 2.01 & 2-Butenoic acid, 2-methyl-, (E)- \\
\hline 8 & 11.01 & 145689 & 0.41 & 24839 & 0.38 & Dodecane, 2,6,11-trimethyl- \\
\hline 9 & 11.29 & 78677 & 0.22 & 17466 & 0.27 & Hexanoic acid \\
\hline 10 & 11.66 & 64866 & 0.18 & 19188 & 0.29 & 1-Heneicosanol \\
\hline 11 & 12.35 & 39374 & 0.11 & 14212 & 0.22 & Heptadecane, 2,6,10,15-tetramethyl- \\
\hline 12 & 12.69 & 106924 & 0.30 & 11102 & 0.17 & 2-Butanone, 4-(2,2,6-trimethylcyclohexyl)- \\
\hline 13 & 13.30 & 356944 & 1.01 & 73595 & 1.12 & Caryophyllene oxide \\
\hline 14 & 13.56 & 91558 & 0.26 & 33046 & 0.50 & Octadecane, 1-chloro- \\
\hline 15 & 13.82 & 291034 & 0.82 & 89776 & 1.37 & 1,6,10-Dodecatrien-3-ol, 3,7,11-trimethyl-, (E)- \\
\hline 16 & 14.66 & 168921 & 0.48 & 78432 & 1.19 & Heneicosane \\
\hline 17 & 14.75 & 79761 & 0.23 & 32959 & 0.50 & $\begin{array}{l}\text { 1H-Cycloprop[e]azulen-7-ol, decahydro-1,1,7-trimethyl-4-methylene-, } \\
\text { [1ar-(1a.alpha.,4a.alpha.,7.beta.,7a.beta.,7b.alpha.)] }\end{array}$ \\
\hline 18 & 14.83 & 408734 & 1.16 & 176706 & 2.69 & 2-Pentadecanone, 6,10,14-trimethyl- \\
\hline 19 & 15.09 & 467380 & 1.32 & 68319 & 1.04 & 1-Heneicosanol \\
\hline 20 & 15.26 & 319379 & 0.90 & 83584 & 1.27 & Caprolactam \\
\hline 21 & 15.67 & 32473 & 0.09 & 18890 & 0.29 & 2-Bromotetradecane \\
\hline 22 & 16.05 & 318485 & 0.90 & 143648 & 2.19 & Selina-6-en-4-ol \\
\hline 23 & 16.09 & 300889 & 0.85 & 133271 & 2.03 & Hexadecanoic acid, ethyl ester \\
\hline 24 & 16.44 & 281577 & 0.80 & 65032 & 0.99 & $\begin{array}{c}\text { 1H-3a,7-Methanoazulene-6-methanol, 2,3,4,7,8,8a-hexahydro-3,8,8-trimethyl-, } \\
\text { [3R-(3.alpha.,3a.beta.,7.beta.,8a.alpha.)] }\end{array}$ \\
\hline 25 & 16.60 & 473246 & 1.34 & 223525 & 3.40 & Heneicosane \\
\hline 26 & 16.79 & 283275 & 0.80 & 117213 & 1.78 & 8-Hydroxy-2,2,8-trimethyldeca-5,9-dien-3-one \\
\hline 27 & 17.49 & 92528 & 0.26 & 39301 & 0.60 & Hexadecane, 2,6,11,15-tetramethyl- \\
\hline 28 & 18.00 & 293141 & 0.83 & 105445 & 1.60 & Eicosanoic acid \\
\hline 29 & 18.19 & 136422 & 0.39 & 55398 & 0.84 & 2-Decene, 2,4-dimethyl- \\
\hline 30 & 18.31 & 447480 & 1.27 & 149416 & 2.27 & 1,6,10-Dodecatrien-3-ol, 3,7,11-trimethyl-, (E)- \\
\hline 31 & 18.51 & 2937254 & 8.31 & 1043306 & 15.88 & Eicosane, 10-methyl- \\
\hline 32 & 18.80 & 151566 & 0.43 & 54379 & 0.83 & 2,6-Octadienal, 2,6-dimethyl-8-(tetrahydro-2H-2-pyranyloxy) \\
\hline 33 & 19.57 & 5322093 & 15.06 & 604327 & 9.20 & Hentriacontane \\
\hline 34 & 20.75 & 11971493 & 33.88 & 1173046 & 17.85 & 1,2-Benzenedicarboxylic acid, diisooctyl ester \\
\hline 35 & 20.89 & 1963448 & 5.56 & 620603 & 9.45 & Heneicosane \\
\hline 36 & 20.97 & 441729 & 1.25 & 151709 & 2.31 & Oxalic acid, 2-ethylhexyl pentadecyl ester \\
\hline 37 & 21.66 & 798268 & 2.26 & 78439 & 1.19 & cis-13,16-Docasadienoic acid \\
\hline 38 & 22.25 & 84015 & 0.24 & 25211 & 0.38 & (2,2,6-Trimethyl-bicyclo[4.1.0]hept-1-yl)-methanol \\
\hline 39 & 22.34 & 448534 & 1.27 & 81446 & 1.24 & Tritetracontane \\
\hline 40 & 22.90 & 2376704 & 6.73 & 483543 & 7.36 & $\begin{array}{l}\text { Cyclopropanemethanol,. alpha.,2-dimethyl-2-(4-methyl-3-pentenyl)-, } \\
\text { [1.alpha.(R*),2.alpha.]- }\end{array}$ \\
\hline \multirow[t]{2}{*}{41} & 23.70 & 925582 & 2.62 & 199546 & 3.04 & Pentadecanoic acid \\
\hline & & 35337195 & 100.00 & 6570408 & 100.00 & \\
\hline
\end{tabular}


TABLE 9: Bioactive constituents of water extract of calyxes of Hibiscus sabdariffa L. from combination treatment of $1000 \mathrm{mg} / \mathrm{L}$ multiwalled carbon nanotubes and $10 \mathrm{ml} / \mathrm{L}$ biostimulators (Delfan plus).

\begin{tabular}{|c|c|c|c|c|c|c|}
\hline & & & & & & Peak Report TIC \\
\hline Peak & R. time & Area & Area\% & Height & Height\% & Name \\
\hline 1 & 5.98 & 179688 & 0.89 & 14717 & 0.29 & 1-[t-Butyl]-3-[2-hydroxyethyl]-2-thiourea \\
\hline 2 & 6.53 & 206765 & 1.03 & 16890 & 0.33 & 1,3,3-Trimethylcyclohex-1-ene-4-carboxaldehyde, $(+,-)$ - \\
\hline 3 & 6.99 & 477865 & 2.38 & 39661 & 0.77 & Bicyclo[2.2.1]heptan-2-one, 1,7,7-trimethyl-, (1S)- \\
\hline 4 & 7.75 & 17402 & 0.09 & 8436 & 0.16 & 2-Octenoic acid, 7-hydroxy-, ethyl ester \\
\hline 5 & 7.99 & 277810 & 1.38 & 25340 & 0.49 & 2-Cyclohexen-1-one, 3-methyl- \\
\hline 6 & 8.72 & 58849 & 0.29 & 11308 & 0.22 & Decanoic acid, 3-methyl- \\
\hline 7 & 8.89 & 61164 & 0.30 & 11322 & 0.22 & 1,6,10-Dodecatriene, 7,11-dimethyl-3-methylene-, (Z)- \\
\hline 8 & 9.38 & 478340 & 2.38 & 65463 & 1.27 & Borneol \\
\hline 9 & 9.84 & 13698 & 0.07 & 7413 & 0.14 & $\begin{array}{c}\text { 1H-Cycloprop[e]azulene, decahydro-1,1,7-trimethyl-4-methylene-, } \\
\text { [1aR-(1a.alpha.,4a.alpha.,7.alpha.,7a.beta.,7b. alpha.)] }\end{array}$ \\
\hline 10 & 9.88 & 11189 & 0.06 & 8044 & 0.16 & $\begin{array}{c}\left.\text { Spiro[cyclopropane- } 1,8^{\prime}\left(1 \mathrm{H}^{\prime}\right)[3 \mathrm{a} .6] \text { methano[3ah] cyclopentacycloocten }\right]-10^{\prime} \text {-one, } \\
\text { octahydro-, (3'as,6'R,9'ar) }\end{array}$ \\
\hline 11 & 10.48 & 861378 & 4.28 & 116784 & 2.27 & 2-Butenoic acid, 2-methyl-, (E)- \\
\hline 12 & 10.99 & 54684 & 0.27 & 15680 & 0.31 & Decane, 2-methyl- \\
\hline 13 & 11.29 & 23403 & 0.12 & 13933 & 0.27 & Heptanoic acid \\
\hline 14 & 11.40 & 96098 & 0.48 & 29717 & 0.58 & 2-Hexanol, 3,3,5-trimethyl-2-(3-methylphenyl)- \\
\hline 15 & 11.62 & 96495 & 0.48 & 30081 & 0.59 & n-Nonadecanol-1 \\
\hline 16 & 12.34 & 43727 & 0.22 & 14425 & 0.28 & Decane, 2-methyl- \\
\hline 17 & 12.58 & 72201 & 0.36 & 17495 & 0.34 & E-6-Octadecen-1-ol acetate \\
\hline 18 & 12.96 & 95214 & 0.47 & 31350 & 0.61 & 1-Dodecanol \\
\hline 19 & 13.30 & 413748 & 2.06 & 114634 & 2.23 & Tricyclo[3.1.0.0(2,4)]hexane, 3,6-diethyl-3,6-dimethyl-, trans- \\
\hline 20 & 13.56 & 58576 & 0.29 & 18742 & 0.37 & 2-Cyclohexene-1-methanol, 2,6,6-trimethyl- \\
\hline 21 & 13.81 & 333618 & 1.66 & 122979 & 2.40 & Nerolidyl acetate \\
\hline 22 & 13.94 & 257018 & 1.28 & 76005 & 1.48 & 2,3-Dipropyl-cyclopropanecarboxylic acid, ethyl ester \\
\hline 23 & 14.00 & 27371 & 0.14 & 18033 & 0.35 & Tetradecanoic acid, ethyl ester \\
\hline 24 & 14.15 & 101642 & 0.51 & 25064 & 0.49 & 10s,11s-Himachala-3(12),4-diene \\
\hline 25 & 14.24 & 103868 & 0.52 & 28501 & 0.56 & 2H-Pyran-2-one, 4-hydroxy-6-methyl- \\
\hline 26 & 14.36 & 65809 & 0.33 & 24124 & 0.47 & Terpin Hydrate \\
\hline 27 & 14.66 & 219672 & 1.09 & 107246 & 2.09 & 2-Bromotetradecane \\
\hline 28 & 14.74 & 136624 & 0.68 & 55418 & 1.08 & $\begin{array}{l}\text { 1H-Cycloprop[e]azulen-7-ol, decahydro-1,1,7-trimethyl-4-methylene-, } \\
\text { [1ar-(1a.alpha.,4a.alpha.,7.beta.,7a.beta.,7b.alpha.)] }\end{array}$ \\
\hline 29 & 14.83 & 943433 & 4.69 & 399599 & 7.78 & 2-Pentadecanone, 6,10,14-trimethyl- \\
\hline 30 & 15.02 & 72288 & 0.36 & 29358 & 0.57 & 2-Piperidinone, 1-(3,4,5,6-tetrahydro-2-pyridinyl)- \\
\hline 31 & 15.11 & 221117 & 1.10 & 85066 & 1.66 & $\begin{array}{l}\text { 7-Oxabicyclo[4.1.0]heptane, } \\
\text { 2,2,6-trimethyl-1-(3-methyl-1,3-butadienyl)-5-methylene- }\end{array}$ \\
\hline 32 & 15.22 & 334667 & 1.66 & 83563 & 1.63 & 2H-Cyclopentacyclooctene, 4,5,6,7,8,9-hexahydro-1,2,2,3-tetramethyl- \\
\hline 33 & 15.34 & 21275 & 0.11 & 13398 & 0.26 & Heptanoic acid, anhydride \\
\hline 34 & 15.82 & 179493 & 0.89 & 59327 & 1.16 & 1H-Cycloprop[e]azulene, decahydro-1,1,7-trimethyl-4-methylene- \\
\hline 35 & 16.04 & 2866546 & 14.25 & 855621 & 16.66 & $\begin{array}{l}\text { 1-Naphthalenol, decahydro-1,4a-dimethyl-7-(1-methylethylidene)-, } \\
\text { [1R-(1.alpha.,4a.beta.,8a.alpha.)]- }\end{array}$ \\
\hline 36 & 16.41 & 159687 & 0.79 & 39983 & 0.78 & 9-Isopropyl-1-methyl-2-methylene-5-oxatricyclo[5.4.0.0(3,8)] undecane \\
\hline 37 & 16.59 & 474369 & 2.36 & 220498 & 4.29 & Tricosane \\
\hline 38 & 16.78 & 432738 & 2.15 & 180735 & 3.52 & Limonen-6-ol, pivalate \\
\hline 39 & 16.96 & 250742 & 1.25 & 76006 & 1.48 & (-)-Spathulenol \\
\hline 40 & 17.12 & 144462 & 0.72 & 59742 & 1.16 & Longipinocarveol, trans- \\
\hline 41 & 17.23 & 116967 & 0.58 & 29430 & 0.57 & 1-Cyclohexyl-1-(2-methylenecyclohexyl)ethanol \\
\hline 42 & 17.39 & 149266 & 0.74 & 44782 & 0.87 & Cyclohexane, butyl- \\
\hline 43 & 17.57 & 118813 & 0.59 & 37221 & 0.72 & (1-Ethylbuta-1,3-dienyl)benzene \\
\hline
\end{tabular}


TABLE 9: Continued.

\begin{tabular}{|c|c|c|c|c|c|c|}
\hline \multicolumn{7}{|r|}{ Peak Report TIC } \\
\hline Peak & R. time & Area & Area\% & Height & Height $\%$ & Name \\
\hline 44 & 17.91 & 52764 & 0.26 & 16286 & 0.32 & Benzeneethanol,. alpha.-ethyl- \\
\hline 45 & 18.00 & 278732 & 1.39 & 107308 & 2.09 & Dodecanoic acid \\
\hline 46 & 18.18 & 203495 & 1.01 & 68303 & 1.33 & But-2-enoic acid, amide, 3-methyl-N-methallyl- \\
\hline 47 & 18.46 & 799323 & 3.97 & 240431 & 4.68 & Heptacosane \\
\hline 48 & 18.65 & 168921 & 0.84 & 74823 & 1.46 & 9,12-Octadecadienoic acid, ethyl ester \\
\hline 49 & 19.46 & 224542 & 1.12 & 39309 & 0.77 & Phytol \\
\hline 50 & 19.92 & 156623 & 0.78 & 28959 & 0.56 & $\begin{array}{l}\text { Acetic acid, } \\
\text { 3-hydroxy-6-isopropenyl-4,8a-dimethyl-1,2,3,5,6,7,8,8a-octahydronaphthalen-2-yl } \\
\text { ester }\end{array}$ \\
\hline 51 & 20.21 & 144167 & 0.72 & 40315 & 0.79 & Oxacycloheptadec-8-en-2-one \\
\hline 52 & 20.31 & 219523 & 1.09 & 59841 & 1.17 & Tetradecanoic acid \\
\hline 53 & 20.50 & 49486 & 0.25 & 17568 & 0.34 & 1,3-Dioxolane, 4,4,5-trimethyl-2-pentadecyl- \\
\hline 54 & 20.60 & 195477 & 0.97 & 54141 & 1.05 & Fumaric acid, ethyl 2-methylallyl ester \\
\hline 55 & 20.80 & 253010 & 1.26 & 76165 & 1.48 & Tetratetracontane \\
\hline 56 & 20.94 & 441843 & 2.20 & 129566 & 2.52 & Oxalic acid, 2-ethylhexyl pentadecyl ester \\
\hline 57 & 21.05 & 123783 & 0.62 & 38025 & 0.74 & Cyclohexane, 1,5-dimethyl-2,3-divinyl- \\
\hline 58 & 21.83 & 1095808 & 5.45 & 63024 & 1.23 & 1-Octacosanol \\
\hline 59 & 22.88 & 1803739 & 8.97 & 328164 & 6.39 & Cyclohexan-1-ethanol, 1-hydroxymethyl- \\
\hline \multirow[t]{2}{*}{60} & 23.70 & 2578313 & 12.82 & 469027 & 9.14 & n-Hexadecanoic acid \\
\hline & & 20119328 & 100.00 & 5134389 & 100.00 & \\
\hline
\end{tabular}

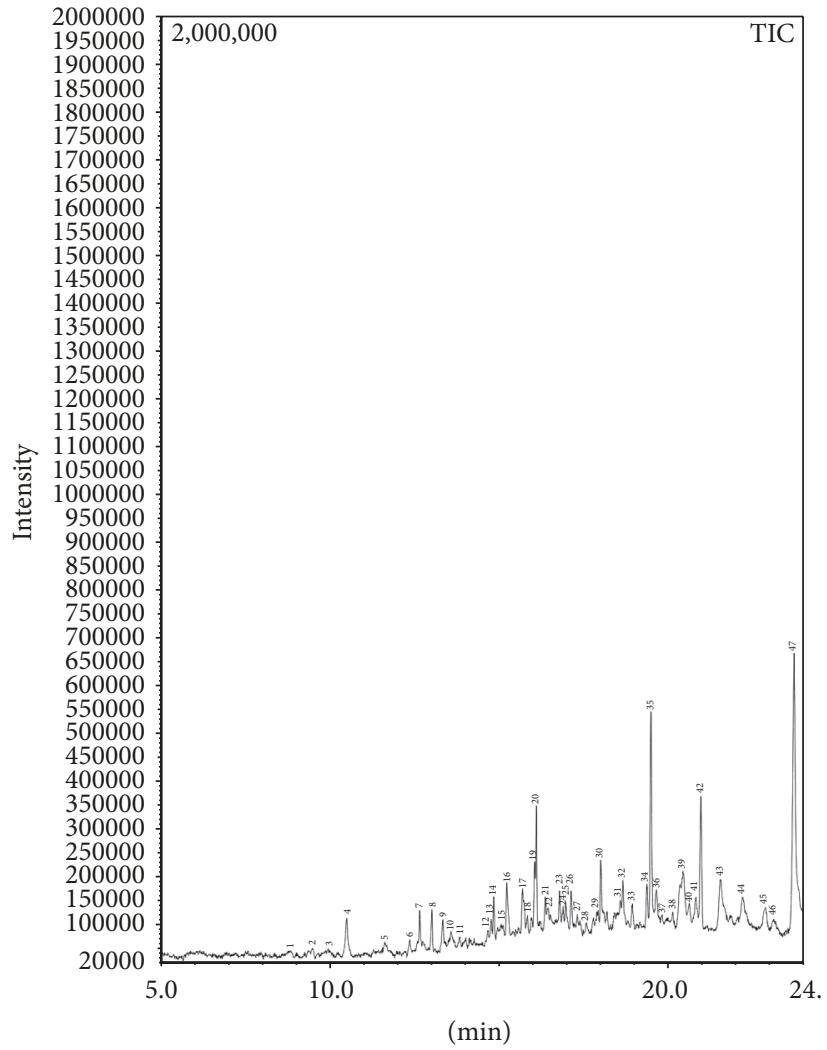

Figure 4: GC-MS chromatogram of water extract of calyxes of karkade (Hibiscus sabdariffa L.) from combination treatment of 500 mg/L multiwalled carbon nanotubes and $10 \mathrm{ml} / \mathrm{L}$ biostimulators (Delfan plus). 


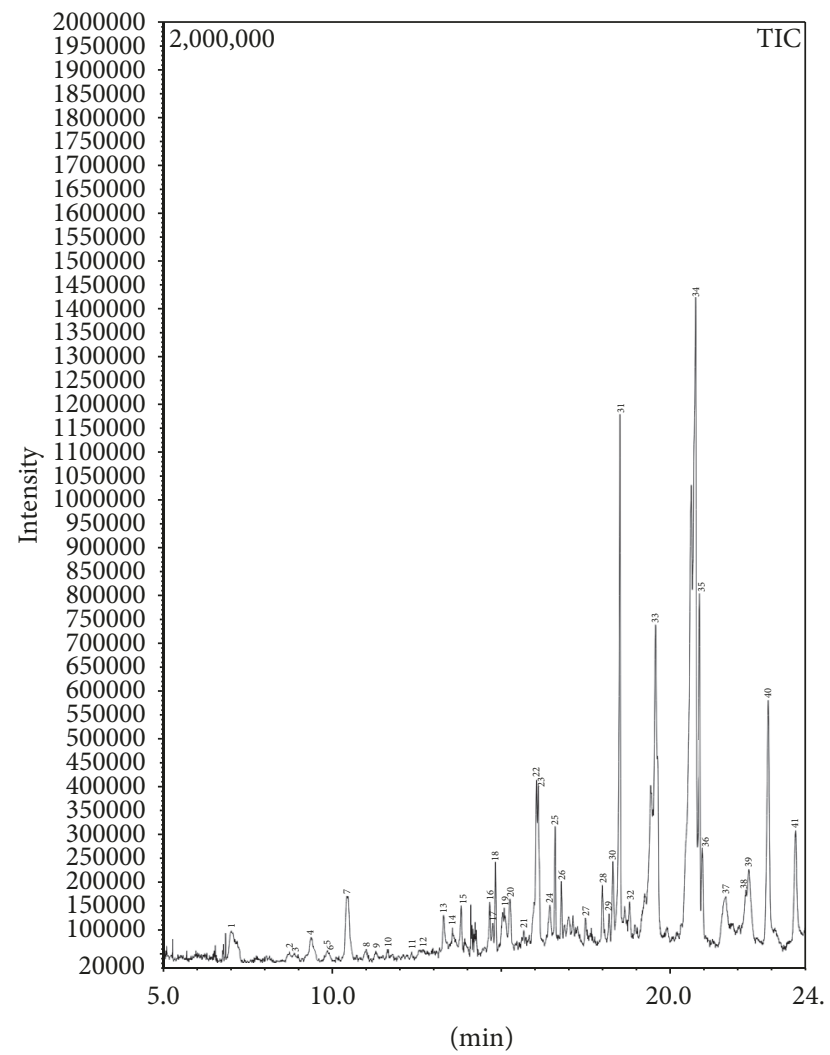

Figure 5: GC-MS chromatogram of water extract of calyxes of karkade (Hibiscus sabdariffa L.) from combination treatment of $1000 \mathrm{mg} / \mathrm{L}$ multiwalled carbon nanotubes and without biostimulators (Delfan plus).

reproduction, and photosynthesis and in response to external signals regulate water channels (aquaporins). There are many reports indicating that aquaporins are central components in plant water relations and are crucial for root water uptake and are a limiting factor for plant growth [19].

Meanwhile, biostimulators (Delfan plus) also caused a significant increase in all studied parameters because they contain free amino acids, total nitrogen, organic matter, and organic carbon (Table 2). These results were in agreement with Saeed et al. [20] on soybean, El-Zohiri and Asfour [21] on potato, and Abo Sedera et al. [22] on strawberry.

Biostimulators act as source of plant growth hormones like gibberellins that could directly or indirectly impact the physiological activities of plant growth and development. The division in the plant with addition of biostimulators leads to increasing the rate of photosynthesis of production of secondary metabolic compounds of plants, particularly in the case of abiotic stress.

Application of exogenous amino acid improves plant growth by increasing the concentration of photosynthetic pigments (ALA is a common precursor to tetrapyrrole) [23, 24], as well as antioxidative enzyme activities [25]. And it affects processes involved in plant nitrogen metabolism (Iwai et al. [26]). Also, amino acids in biostimulant have positive effects on plant growth and yield and significantly relieve the injuries caused by abiotic stresses [8]; the addition of

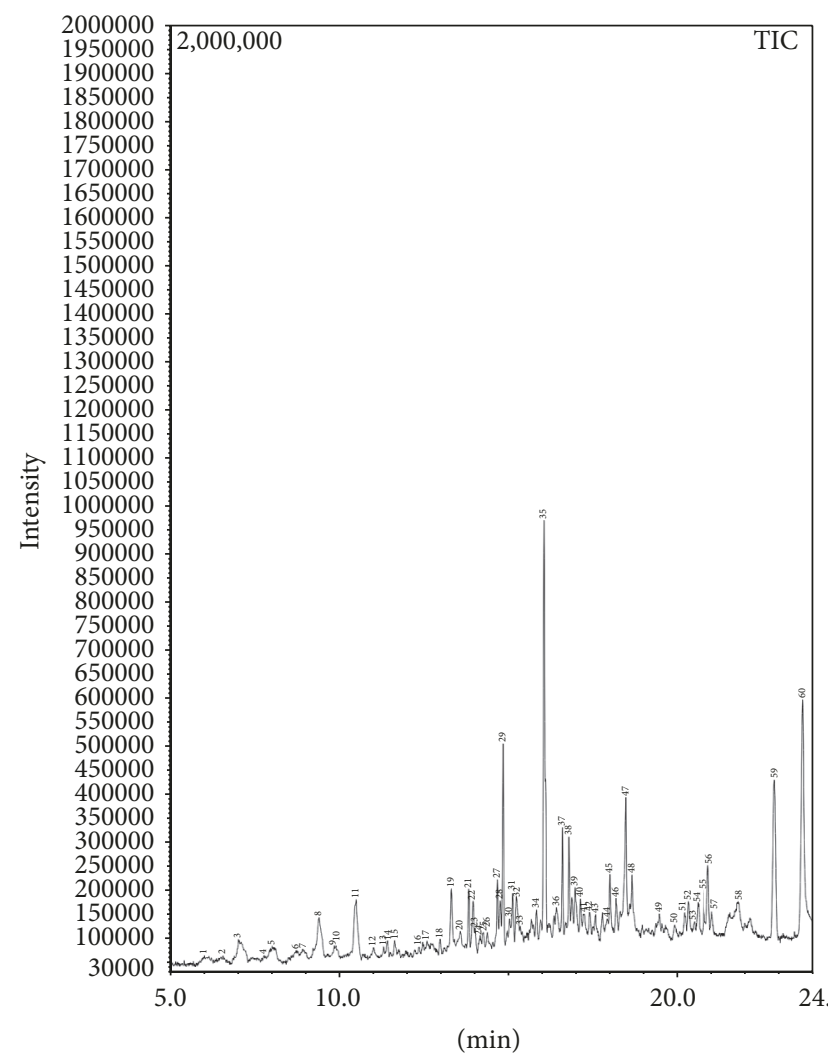

FIGURE 6: GC-MS chromatogram of water extract of calyxes of Hibiscus sabdariffa L. from combination treatment of $1000 \mathrm{mg} / \mathrm{L}$ multiwalled carbon nanotubes and $10 \mathrm{ml} / \mathrm{L}$ biostimulators (Delfan plus).

high concentration of multiwalled carbon nanotubes to plant probably causes stress to the plant.

\section{Conclusions}

Data from results suggest that experimental factors have an important role in causing quantitative and qualitative variations of their medically active ingredients compared with untreated plants. Combination treatment of $1000 \mathrm{mg} / \mathrm{L}$ multiwalled carbon nanotubes and $10 \mathrm{ml} / \mathrm{L}$ biostimulators (Delfan plus) caused an increase in all studied parameters of karkade.

\section{Conflicts of Interest}

The author declares that they have no conflicts of interest.

\section{References}

[1] E. O. Odebunmi, O. O. Dosunmu, and E. A. Jedede, "Biophysico-chemical Analysis and Fermentation Studies of Hibiscus Sabdariffa," NJS, vol. 30, no. 1, pp. 1-8, 2002.

[2] M. M. Ramirez-Rodrigues, M. L. Plaza, A. Azeredo, M. O. Balaban, and M. R. Marshall, "Physicochemical and phytochemical properties of cold and hot water extraction from 
Hibiscus sabdariffa," Journal of Food Science, vol. 76, no. 3, pp. C428-C435, 2011.

[3] V. Hirunpanich, A. Utaipat, N. P. Morales et al., "Antioxidant effects of aqueous extracts from dried calyx of hibiscus sabdariffa Linn. (roselle) in vitro using rat low-density lipoprotein (LDL)," Biological \& Pharmaceutical Bulletin, vol. 28, no. 3, pp. 481-484, 2005.

[4] M. T. Olaleye, "Cytotoxicity and antibacterial activity of methanolic extract of Hibiscus sabdariffa," J Med Plants Res, vol. 1, pp. 9-13, 2007.

[5] N. M. Nnam and N. G. Onyeke, "Chemical composition of two varieties of sorrel (Hibiscus sabdariffa L.), calyces and the drinks made from them," Plant Foods for Human Nutrition, vol. 58, no. 3, pp. 1-7, 2003.

[6] I. Da-Costa-Rocha, B. Bonnlaender, H. Sievers, I. Pischel, and M. Heinrich, "Hibiscus sabdariffa L.-a phytochemical and pharmacological review," Food Chemistry, vol. 165, pp. 424-443, 2014.

[7] M. V. Khodakovskaya, K. De Silva, D. A. Nedosekin et al., "Complex genetic, photothermal, and photoacoustic analysis of nanoparticle-plant interactions," Proceedings of the National Acadamy of Sciences of the United States of America, vol. 108, no. 3, pp. 1028-1033, 2011.

[8] K. Kowalczyk and T. Zielony, "Effect of Aminoplant and Asahi on yield and quality of lettuce grown on rockwool," in Proceedings of the Conference of biostimulators in modern agriculture, Warsaw, Poland, 2008.

[9] K. Srinivasan, S. Sivasubramanian, and S. Kumaravel, "Phytochemical profiling and GCMS study of Adhatoda vasica leaves," International Journal of Pharma and Bio Sciences, vol. 5, no. 1, pp. B714-B720, 2014.

[10] R. G. D. Steel and J. H. Torrie, Principles and Procedures of Statistics, McGraw- Hill Book Co., New York, NY, USA, 2nd edition, 1980.

[11] J. E. Canas, M. Long, S. Nations et al., "Effects of functionalized and nonfunctionalized single-walled carbon nanotubes on root elongation of select crop species," Environmental Toxicology and Chemistry, vol. 27, no. 9, pp. 1922-1931, 2008.

[12] M. Khodakovskaya, E. Dervishi, M. Mahmood et al., "Carbon nanotubes are able to penetrate plant seed coat and dramatically affect seed germination and plant growth," ACS Nano, vol. 3, no. 10, pp. 3221-3227, 2009.

[13] H. R. Heydari, A Study on Application of Carbon Nanotubes (CNTs) as a Plant Growth Regulator in Anthurinium andreanum L. Micropropagation [M. S. thesis], University of Tarbiat Modares, 2013.

[14] M. V. Khodakovskaya, K. De Silva, A. S. Biris, E. Dervishi, and H. Villagarcia, "Carbon nanotubes induce growth enhancement of tobacco cells," ACS Nano, vol. 6, no. 3, pp. 2128-2135, 2012.

[15] A. Husen and K. S. Siddiqi, "Carbon and fullerene nanomaterials in plant system," Journal of Nanobiotechnology, vol. 12, no. 16, pp. 1-10, 2014.

[16] R. A. Taha, "Nanotechnology and its application in agriculture," Advances in Plants Agriculture Research, vol. 3, no. 2, p. 00089 , 2016.

[17] C. M. Rico, S. Majumdar, M. Duarte-Gardea, J. R. Peralta-Videa, and J. L. Gardea-Torresdey, "Interaction of nanoparticles with edible plants and their possible implications in the food chain," Journal of Agricultural and Food Chemistry, vol. 59, no. 8, pp. 3485-3498, 2011.
[18] S. J. Klaine, P. J. J. Alvarez, G. E. Batley et al., "Nanomaterials in the environment: behavior, fate, bioavailability, and effects," Environmental Toxicology and Chemistry, vol. 27, no. 9, pp. 1825$1851,2008$.

[19] R. Kaldenhoff and M. Fischer, "Aquaporins in plants," Acta Physiologica, vol. 187, no. 1-2, pp. 169-176, 2006.

[20] M. R. Saeed, A. M. Kheir, and A. A. Al-Sayed, "Supperssive effect of some amino acids against Meloidogyne incognita on Soybeans.J", Agric. Sci. Mansoura Univ, vol. 30, no. 2, pp. 10971103, 2005.

[21] S. S. M. El-Zohiri and Y. M. Asfour, "Effect of some organic compounds on growth and productivity of some potato cultivars," Annals of Agriculture Science, vol. 47, no. 3, pp. 403-415, 2009.

[22] F. Abo Sedera, A. Amany, A. Abd El-Latif, L. A. A. Bader, and S. M. Rezk, "Effect of NPK mineral fertilizer levels and foliar application with humic and amino acids on yield and quality of strawberry," Egyp.J. of Appl. Sci, pp. 25-154, 2010.

[23] T. Tanaka, K. Iwai, K. Watanabe, and Y. Hotta, "Development of 5 -aminolevulinic acid for agriculture uses," Regul. Plant Growth Devel, vol. 40, no. 1, pp. 22-29, 2005.

[24] E. Yaronskaya, I. Vershilovskaya, Y. Poers, A. E. Alawady, N. Averina, and B. Grimm, "Cytokinin effects on tetrapyrrole biosynthesis and photosynthetic activity in barley seedlings," Planta, vol. 224, no. 3, pp. 700-709, 2006.

[25] S. A. Memon, X. Hou, L. Wang, and Y. Li, "Promotive effect of 5aminolevulinic acid on chlorophyll, antioxidative enzymes and photosynthesis of Pakchoi (Brassica campestris ssp. chinensis var. communis Tsen et Lee)," Acta Physiologiae Plantarum, vol. 31, no. 1, pp. 51-57, 2009.

[26] K. Iwai, A. Saito, J. Van Leeuwen, T. Tanaka, and Y. Takeuchi, "A new functional fertilizer containing 5 -aminolevulinic acid promoted hydroponically-grown vegetables in the Netherlands," Acta Horticulturae, vol. 697, pp. 351-355, 2005. 


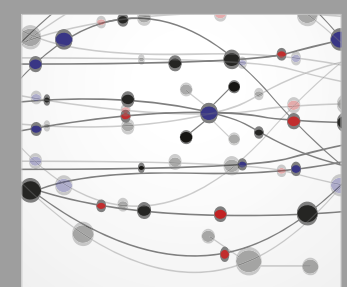

The Scientific World Journal
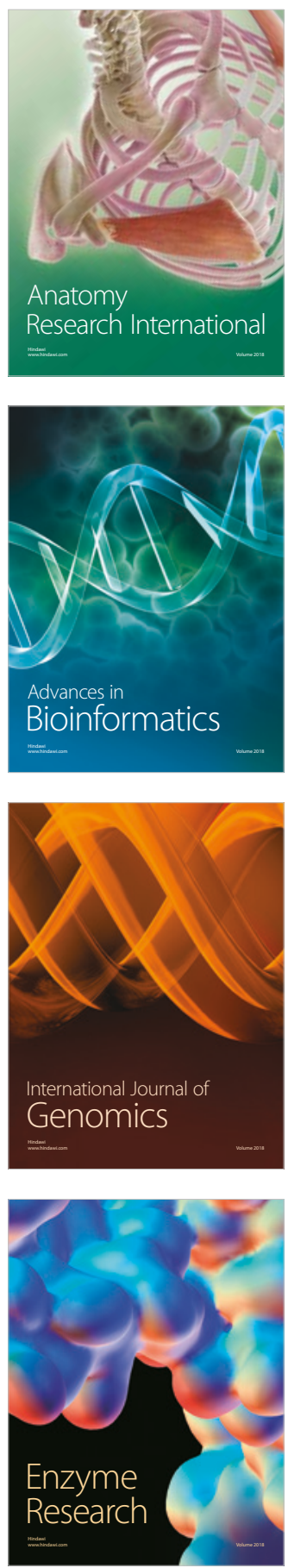
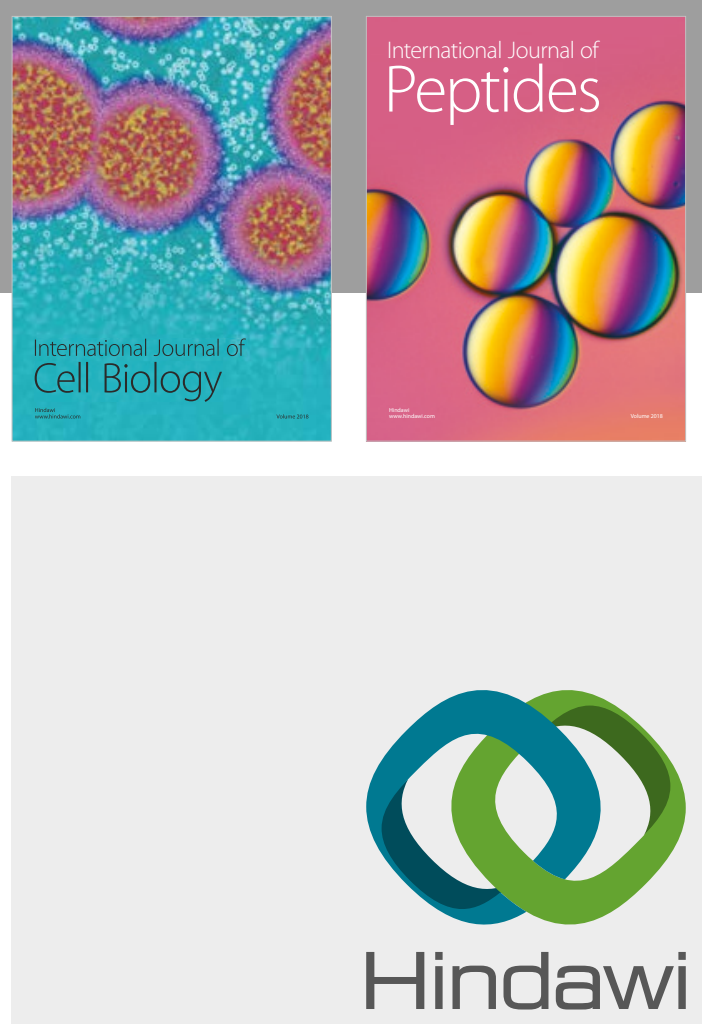

Submit your manuscripts at

www.hindawi.com
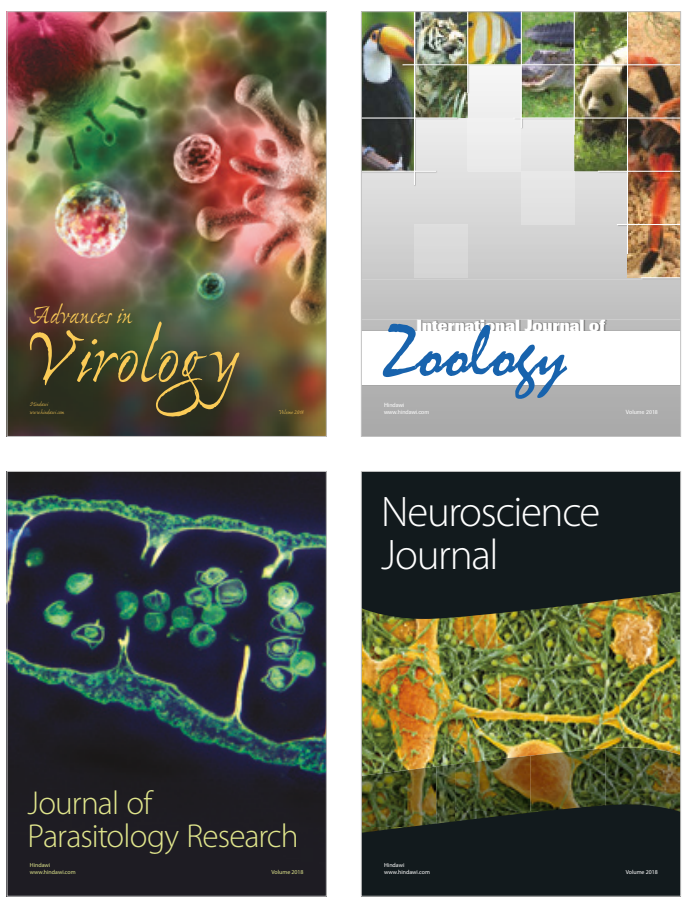
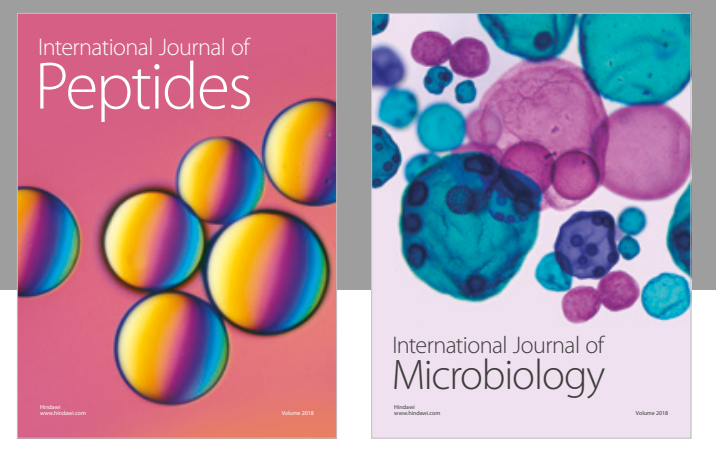

nternational Journal of Microbiology
Journal of
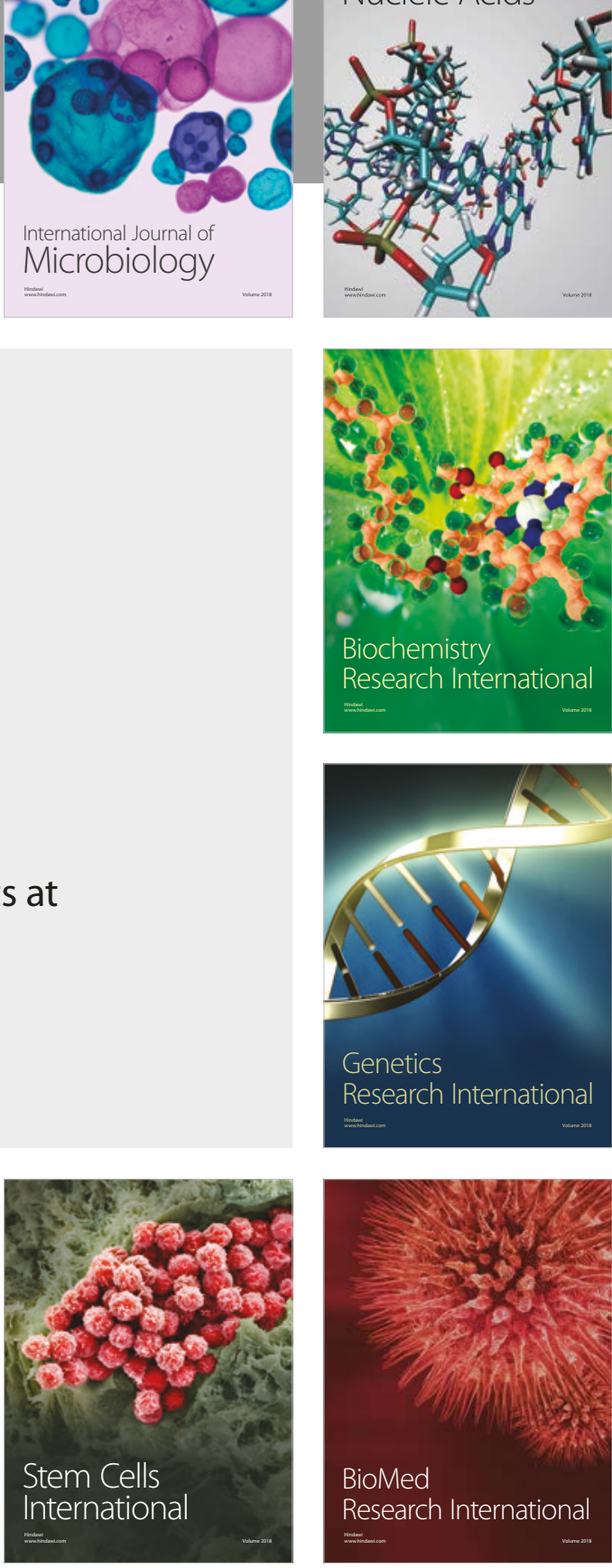
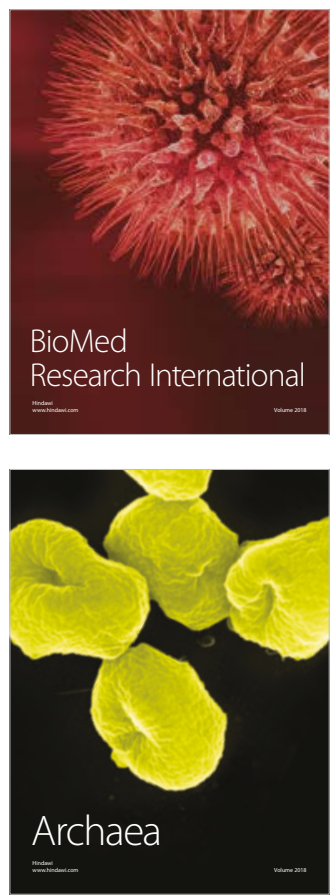